\title{
MATHEMATICAL MODEL OF DENGUE CONTROL WITH CONTROL OF MOSQUITO LARVAE AND MOSQUITO AFFECTED BY CLIMATE CHANGE
}

\author{
Mohammad Soleh ${ }^{1}$, Wartono $^{2}$, Yuslenita Muda ${ }^{3 *}$ \\ ${ }^{1,2,3}$ Department of Mathematics, Faculty of Science and Technology, \\ State Islamic University of Sultan Syarif Kasim Riau \\ Jl. HR. Soebrantas No.155 Simpang Baru, Panam, Pekanbaru, 28293 \\ Corresponding author email: ${ }^{3 *}$ yuslenita.muda@uin-suska.ac.id
}

\begin{abstract}
Consider a SIR model for the spread of dengue hemorrhagic fever involving three populations, mosquito eggs, mosquitoes, and humans. The parameters of the SIR model were estimated using rainfall data and air temperature for the cities of Pekanbaru and Solok. The main aim of this paper is to determine the effect of mosquito larvae and adult mosquito control on the spread of the dengue virus. Numerical solutions were also presented by using the Runge-Kutta method of order 4. Based on the results, the SIR model was obtained by involving the control parameters of mosquito larvae and adult mosquitoes. Besides, the mosquito population is affected by changes in temperature, rainfall, and fog. Numerical simulations illustrate that the number of infected mosquitoes and infected humans is influenced by the parameters of the percentage of mortality of mosquito larvae and adult mosquitoes.
\end{abstract}

Keywords: Dengue Fever, Climate Change, SIR Model, Rainfall 


\section{INTRODUCTION}

Till recently, Dengue Hemorrhagic Fever (DHF) still ranks at the top for infectious diseases in Pekanbaru and Solok. Every week there are always DHF patients who are treated at the general practitioner (puskesmas) or hospital. The difficulty in reducing dengue cannot be separated from the many factors that are suspected to be the trigger. Climatic factors (rainfall, temperature, humidity), population density, and level of public awareness to maintain environmental sanitation are thought to be the main factors triggering DHF cases. Another factor is the intrinsic factor of the Aedes Aegypti mosquito itself which is classified as very resilient [1]. The influence of climate on the number of DHF cases can be seen in the number of sufferers during the rainy season compared to the dry season. In the rainy season but warm temperatures are ideal conditions for mosquitoes to have optimum oviposition, egg maturity is more perfect, the virus incubation period is shorter. The impact is that the number of mosquitoes is abundant and sufferers increase [2], [3].

DHF is not transmitted directly from human to human. Therefore, handling dengue fever can be done by breaking the chain of virus carriers by reducing as much as possible the Aedes Aegypti mosquito. These efforts include eradicating mosquito nests program, chemically killing larvae, and using predators such as larvae eating fish and cyclops. Supriatna [4] recommends continuously exterminating larvae to reduce the number of mosquitoes. However, this effort is not easy considering that the Aedes Aegypti mosquito's survival rate is quite high. Mosquito eggs can last up to 2 years until they meet the water and then hatch. When conditions are dry, mosquitoes can survive between rocks or in the bush. Then, when the larvae grow in abundance, a female mosquito will lay up to 300-600 eggs. Besides, mosquitoes can move places following human mobility [1].

This paper examines the control factors given to the model. Thus, there are natural factors that trigger the number of DHF, namely climate (rainfall and temperature) and factors are controlling DHF. The result to be taken into account is how the effect of control on larvae and adult mosquitoes can reduce cases of dengue affected by climate. The results will be described as the number of individuals infected with DHF during climate change and control treatment. Several studies on DHF that can be referenced have been carried out by [5], [4]. The study of dengue fever which is related to temperature changes was carried out by [6], [7], [8], and [9]. The study of DHF associated with changes in rainfall was done by [10]. The study of DHF associated with changes in temperature and rainfall was done by [2], [3]. The consider of DFH related with control variables was carried out by [11], [12], [13], [14], [15]. All of these studies use mathematical models and take case studies in a particular place.

\section{RESEARCH METHOD}

The research method used in this research is a literature study related to DHF, especially mathematical models with the influence of climate and control of larvae and mosquitoes. Then by estimating the parameters from the DHF patient data and climate (temperature-rainfall) in Pekanbaru City and Solok City, the model will be stimulated to produce an overview of the spread of DHF in the two cities.

\subsection{Differential Equations System}

A differential equation is an equation that involves the derivative of one or more dependent variables to one or more independent variables, while the system of differential equations consists of several differential equations. Given a system of differential equations as follow

$$
\begin{gathered}
\dot{x_{1}}=f_{1}\left(x_{1}, x_{2}, \ldots, x_{n}\right), \\
\dot{x_{2}}=f_{2}\left(x_{1}, x_{2}, \ldots, x_{n}\right), \\
\vdots \\
\dot{x_{n}}=f_{n}\left(x_{1}, x_{2}, \ldots, x_{n}\right),
\end{gathered}
$$

with $E \subset R^{n}$, and $\boldsymbol{f}: E \rightarrow R^{n}$ continuous function on $E$. System (1) can be written as

$$
\dot{x}=f(x) .
$$


If $f_{1}, f_{2}, \ldots, f_{n}$ linear function in $x_{1}, x_{2}, \ldots, x_{n}$ respectively, then system (2) is called a system of linear differential equations which can be written as

$$
\begin{gathered}
\dot{x_{1}}=a_{11} x_{1}+a_{12} x_{2}+a_{13} x_{3}+\cdots+a_{1 n} x_{n} \\
\dot{x_{2}}=a_{21} x_{1}+a_{22} x_{2}+a_{23} x_{3}+\cdots+a_{2 n} x_{n} \\
\vdots \\
\dot{x_{n}}=a_{n 1} x_{1}+a_{n 2} x_{2}+a_{n 3} x_{3}+\cdots+a_{n n} x_{n} .
\end{gathered}
$$

System (3) can be expressed in the form $\dot{x}=A x$, with $x \in E$ and $A$ is $n \times n$ matrix. System (2) is called a nonlinear system if it cannot be expressed in the form of a System (3).

\subsection{SIR Model of Dengue Fever Spread without Climate Effect}

Lourdes Esteva and Cristobal Vargas were the first to use the SIR model to analyze the spread of DHF. In the case of DHF, there are two populations, namely the human population and the mosquito (vector) population. In the human population, there are three sub-classes, namely the suspectable class or class containing individuals susceptible to dengue disease, the infectives class or class containing individuals infected with dengue and can transmit the disease, and the Recovery class, which is a class containing individuals who have recover and have permanent immunity against dengue disease. If $N_{H}(t)$ states the number of humans at time $t, S_{H}(t)$ states the number of individuals who suspect at time $t, I_{H}(t)$ states the number of individuals infected at time $t$, and $R_{H}(t)$ states the number of individuals recovered at time $t$, then

$$
N_{H}(t)=S_{H}(t)+I_{H}(t)+R_{H}(t)
$$

Whereas in the mosquito population there are only two sub-classes, namely the Suspectable Vector class and the Infectible Vector class. There is no cure class for mosquitoes considering the relatively short life span of mosquitoes. Furthermore, with $S_{V}(t)$ stating the number of suspect mosquitoes at time $t, I_{V}(t)$ states the number of infected mosquitoes at time $t$, and $N_{V}(t)$ states the total number of mosquitoes, then the mathematical relationship can be written as:

$$
N_{V}(t)=S_{V}(t)+I_{V}(t)
$$

The following is the flow chart for the DHF distribution model by Esteva-Vargas:

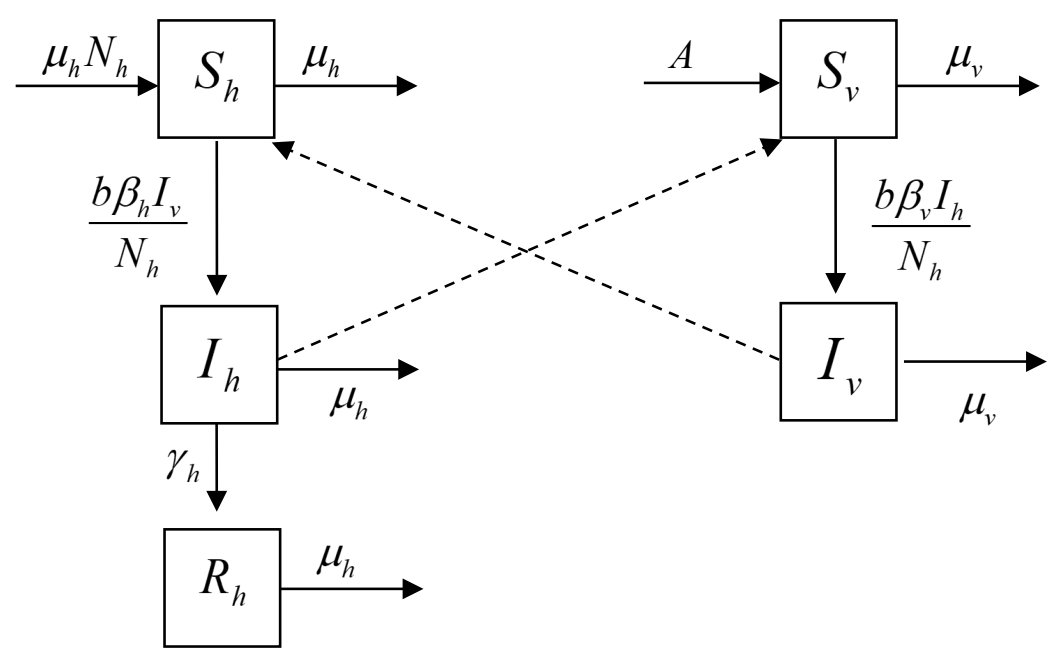

Scheme 1. DHF distribution model

with $A$ represents the rate of addition of mosquitoes (recruitment rate), $\mu_{h}$ is the birth and death rate, $b$ is the average mosquito bite, is the rate of transmission through infected mosquito bites, $\beta_{h}$ is the rate of transmission through infected mosquito bites, $\gamma_{h}$ is the rate of humans recovering from illness, $\mu_{v}$ is the mosquito death rate, and $\beta_{h}$ is the rate of transmission through infected mosquito bites, $\beta_{v}$ the transmission rate from infected humans who are bitten by mosquitoes.

The SIR model for the spread of DHF proposed by Esteva-Vargas is as follows: 


$$
\begin{aligned}
& \frac{d S_{H}}{d t}=\mu_{H} N_{H}-\frac{\beta_{H} k}{N_{H}} S_{H} I_{V}-\mu_{H} S_{H} \\
& \frac{d I_{H}}{d t}=\frac{\beta_{H} k}{N_{H}} S_{H} I_{V}-\mu_{H} I_{H}-\gamma_{H} I_{H} \\
& \frac{d R_{H}}{d t}=\gamma_{H} I_{H}-\mu_{H} R_{H} \\
& \frac{d S_{V}}{d t}=a N_{V}-\frac{\beta_{V} k}{N_{H}} S_{V} I_{H}-\mu_{V} S_{V} \\
& \frac{d I_{V}}{d t}=\frac{\beta_{V} k}{N_{H}} S_{V} I_{H}-\mu_{V} I_{V} .
\end{aligned}
$$

\section{RESULT AND DISCUSSION}

\subsection{Establishment of a SIR Model with the influence of climate change}

This study adopted a model from Chen, et.al, Morgan Rossi, et.al, Supriatna, et.al [8], and Syafarudin et.al [9] to study the spread of DHF in Pekanbaru and Solok City using the SIR model. Some of the assumptions for the human population in this study include:

a. DHF transmission occurs when a mosquito infected with the virus bites a healthy person and /or healthy mosquito bites an infected person.

b. In a population, there is a process of birth and a process of death with an exponential growth rate.

Continuing the model from Esteva-Vargas, for the population of mosquito eggs/larvae, there are mosquito eggs/larvae that are still healthy (susceptible) and there are mosquito eggs/larvae contaminated with the dengue virus due to vertical infection from infected female mosquitoes. If $N_{e}$ states the total number of mosquito eggs/larvae produced by adult mosquitoes, $S_{e}$ is the number of healthy mosquito eggs/ larvae, and $I_{e}$ states the number of mosquito eggs/larvae infected with dengue, then it is obtained:

$$
N_{e}=S_{e}+I_{e} .
$$

In the $S_{e}$ class, there was an increase in the number of healthy eggs/larvae due to the presence of adult female mosquitoes that lay eggs/larvae. But the number is reduced because some of the eggs/larvae hatch and some also die. The $I_{e}$ class increases from the eggs/larvae that come out of infected female mosquitoes and decreases due to the eggs/larvae that have been cooked/hatched and which are dead. With $k_{1}$ stating the percentage of death of mosquito eggs/larvae due to drug administration, the $S_{e}$ and $I_{e}$ correlations can be formulated as follows:

$$
\begin{aligned}
& \frac{d S_{e}}{d t}=b\left(1-v\left(\frac{I_{v}}{S_{v}+E_{v}+I_{v}}\right)\right)-\left(1+k_{1}\right) \mu_{e} S_{e}+\left(1-k_{1}\right) \omega S_{e}, \\
& \frac{d I_{e}}{d t}=b v\left(\frac{I_{v}}{S_{v}+E_{v}+I_{v}}\right)-\left(1+k_{1}\right) \mu_{e} I_{e}-\left(1-k_{1}\right) \omega I_{e},
\end{aligned}
$$

with $b, v, \omega$, and $\mu_{e}$ represent the oviposition rate of female mosquitoes, the proportion of vertical infection incidence of adult female mosquitoes to eggs/larvae, rate of hatching of larvae of mosquitoes into larvae, and mortality rates of mosquito larvae respectively.

In the adult (female) mosquito population, there are three classes, namely the susceptible mosquito population class, the exposed mosquito population class, and the infected mosquito population class. Mathematically, the three population classes can be formulated as follows:

$$
\begin{aligned}
& \frac{d S_{v}}{d t}=\left(1-k_{1}\right) \omega S_{e}-\beta \frac{I_{h}}{N_{h}} S_{v}-\left(\mu_{v}+k_{2}\right) S_{v}, \\
& \frac{d E_{v}}{d t}=\beta \frac{I_{h}}{N_{h}} S_{v}-\varepsilon E_{v}-\left(\mu_{v}+k_{2}\right) E_{v}, \\
& \frac{d I_{v}}{d t}=\varepsilon E_{v}+\left(1-k_{1}\right) \omega I_{e}-\left(\mu_{v}+k_{2}\right) I_{v},
\end{aligned}
$$


with $\varepsilon, \mu_{v}, k_{2}$ successively represent the transmission rate of exposed mosquitoes to become infected, the natural mortality rate of mosquitoes, and the percentage of adult mosquito deaths due to fogging.

Thus, based on (6) - (8) the SIR model for the spread of DHF with the effect of climate change is obtained as follows:

$$
\begin{aligned}
& \frac{d S_{e}}{d t}=b\left(1-v\left(\frac{I_{v}}{S_{v}+E_{v}+I_{v}}\right)\right)-\left(1+k_{1}\right) \mu_{e} S_{e}+\left(1-k_{1}\right) \omega S_{e} \\
& \frac{d I_{e}}{d t}=b v\left(\frac{I_{v}}{S_{v}+E_{v}+I_{v}}\right)-\left(1+k_{1}\right) \mu_{e} I_{e}-\left(1-k_{1}\right) \omega I_{e} \\
& \frac{d S_{v}}{d t}=\left(1-k_{1}\right) \omega S_{e}-\beta \frac{I_{h}}{N_{h}} S_{v}-\left(\mu_{v}+k_{2}\right) S_{v} \\
& \frac{d E_{v}}{d t}=\beta \frac{I_{h}}{N_{h}} S_{v}-\varepsilon E_{v}-\left(\mu_{v}+k_{2}\right) E_{v} \\
& \frac{d I_{v}}{d t}=\varepsilon E_{v}+\left(1-k_{1}\right) \omega I_{e}-\left(\mu_{v}+k_{2}\right) I_{v} \\
& \frac{d S_{h}}{d t}=\mu_{h} N_{h}-\frac{\beta}{N_{h}} S_{h} I_{v}-\mu_{h} S_{h} \\
& \frac{d I_{h}}{d t}=\beta \frac{S_{h}}{N_{h}} I_{v}-\gamma_{h} I_{h}-\mu_{h} I_{h} \\
& \frac{d R_{h}}{d t}=\gamma_{h} I_{h}-\mu_{h} R_{h} .
\end{aligned}
$$

\subsection{Climate as an Independent Variable of Entomological Parameters}

There are five entomological parameters related to the spread of DHF in this study, namely: oviposition rate of mosquito eggs/larvae, mosquito egg/larva mortality, hatching rate of eggs/mosquito larvae, adult mosquito mortality rate, dengue virus incubation rate, and transmission rate. These six parameters can be expressed as a function of temperature, rainfall, or both.

a. The oviposition rate of mosquito eggs/larvae $(b)$

$$
b(T, R)=0,0011\left[1-\exp (0,0101(T-40))(T-9)^{3,445}\right]\left[\left[\frac{0,0392}{1+2,11 \frac{\left(R-30,99^{2}\right.}{28,218}}\right]^{0,737}+0,126\right]
$$

b. Mosquito egg/larva mortality rate

$$
\mu_{\mathrm{e}}=0,0731 \exp (0,0595 T)
$$

c. Hatching rate of eggs/larvae

$$
\omega(T)=-0.0000002 T^{5}+0.00003 T^{4}-0.0012 T^{3}+0.0248 T^{2}-0.2464 T+0.9089
$$

d. The mortality rate for adult female mosquitoes

e. Dengue virus incubation parameters

$$
\mu_{v}(T)=p_{19} \exp \left(-p_{20}\left(T-p_{21}\right)^{2}+p_{22}\left(T-p_{21}\right)^{2}\right.
$$

f. Transmission rate

$$
\varepsilon(T)=p_{23}-p_{24}[\ln (T)]^{2} .
$$

$$
\beta(T)=\frac{0,03 T-0,66}{7}\left(0.001044 \cdot T \cdot(T-12.286) \cdot(32.461-T)^{\frac{1}{2}}\right)
$$

The results of parameter estimation based on data of temperature, rainfall, and the number of DHF sufferers, cases of DHF recovery can be seen in Table 1, below: 
Table 1. Entomological parameters and host population

\begin{tabular}{crrr}
\hline Parameters & Pekanbaru & \multicolumn{1}{c}{ Solok } & Information \\
\hline$v$ & 0,028 & 0,028 & S-C Chen, et.al \\
\hline$\beta$ & 0,02076941776 & 0,01255547746 & Estimation \\
\hline$\omega$ & 1,7891486 & 1,31076641 & Estimation \\
\hline$b$ & 0,3614758369 & 0,2840196049 & Estimation \\
\hline$\varepsilon$ & 0,2955969909 & 0,3402025690 & Estimation \\
\hline$\mu_{v}$ & 0,006469390419 & 0,01743452765 & Estimation \\
\hline$\mu_{e}$ & 0,3644277688 & 0,3274149631 & Estimation \\
\hline$\gamma$ & 0,0007690192 & 0,001424585 & Central Bureau of Statistics \\
\hline$\mu_{h}$ & 0,0249 & 0,0199 & Central Bureau of Statistics \\
\hline$N_{h}$ & 1.091 .088 & 68.608 & Central Bureau of Statistics \\
\hline$k_{1}$ & 0,80 & 0,80 & Assumption \\
\hline$k_{2}$ & 0,75 & 0,75 & Assumption \\
\hline$N_{v}$ & 1.000 .000 & 200.000 & Assumption \\
\hline$S_{h, 0}$ & 1.091 .083 & 68.607 & Public Health Office \\
\hline$I_{h, 0}$ & 15 & 10 & Public Health Office \\
\hline$R_{h, 0}$ & 14 & 9 & Public Health Office \\
\hline$S_{e, 0}$ & 5.000 & 350 & Assumption \\
\hline$I_{e, 0}$ & 1.000 .000 & 25 & Assumption \\
\hline$S_{v, 0}$ & 50.000 & Assumption \\
\hline$E_{v, 0}$ & 100.000 & 5.000 & Assumption \\
\hline$I_{v, 0}$ & 5.000 & Assumption \\
\hline
\end{tabular}

\subsection{Numerical Simulations}

As an illustration of the changes in $S_{v}, I_{v}$, and $E_{v}$ with respect to $t$ for Pekanbaru and Solok city respectively, are given in Figures 1 and 2 below.

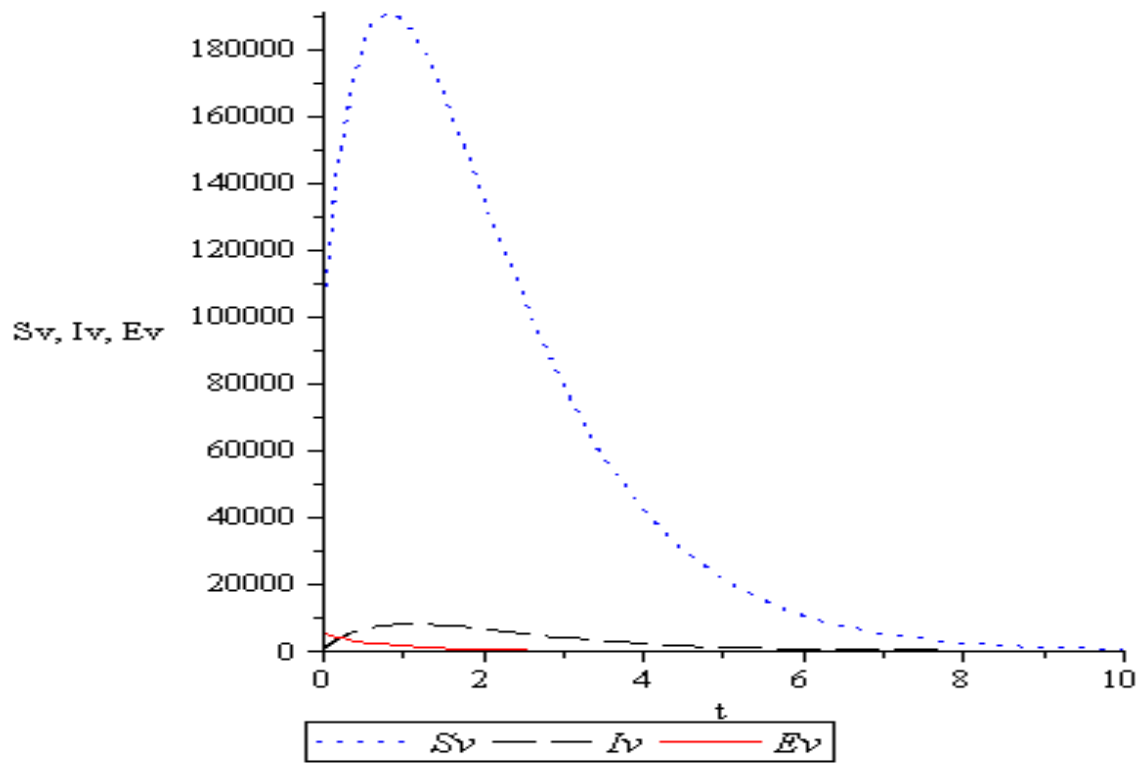

Figure 1. The graph of $S_{v}, I_{v}$ and $E_{v}$ with respect to $t$ for $k_{1}=0.8$ and $k_{2}=0.8$ in the case of Solok City 


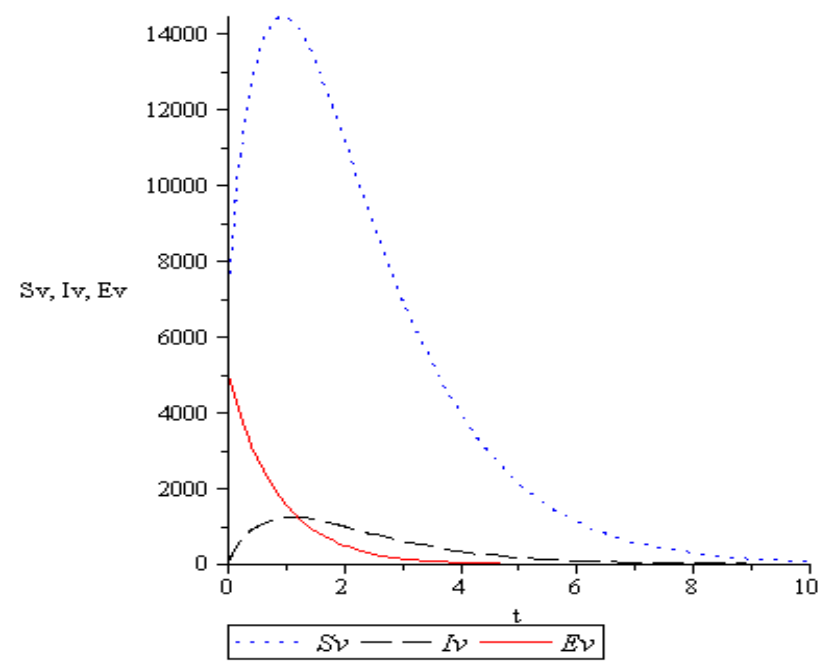

Figure 2. The graph of $S_{v}, I_{v}$ and $E_{v}$ with respect to $t$ for $k_{1}=0.8$ and $k_{2}=0.8$ in the case of Solok City

In this paper, we involve two control parameters, namely the percentage of mosquito larvae mortality $\left(k_{1}\right)$ and the percentage of adult mosquito mortality $\left(k_{2}\right)$. The eradication of mosquito larvae is carried out by using abate powder which is spread or sprinkled on the breeding places for mosquito larvae, while adult mosquito eradication is carried out by using fogging on places that have the potential to become adult mosquito nests.

Figures 3 and 4 below show the effect of eradicating adult mosquitoes on the development of the number of mosquitoes that have the potential to spread DHF.

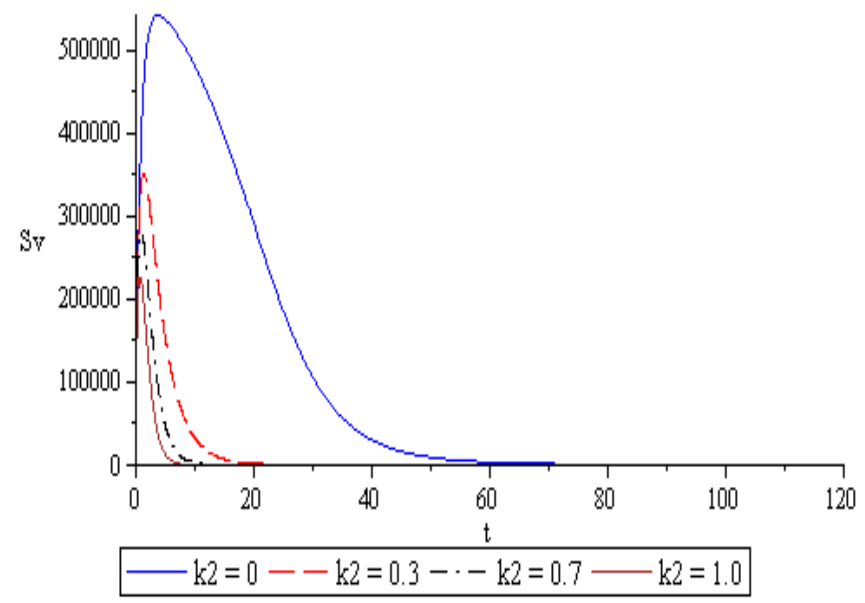

Figure 3. The graph of $S_{v}$ with respect to $t$ for $k_{1}=0.7$ in the case of Pekanbaru City

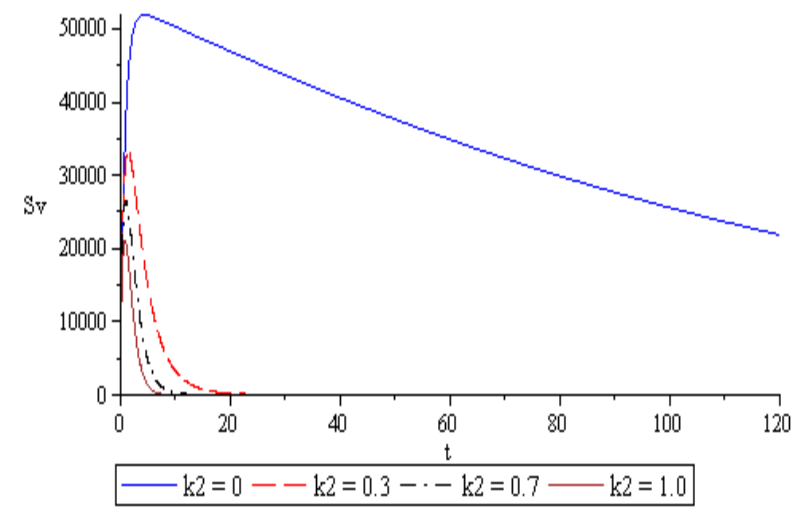

Figure 4. The graph of $S_{v}$ with respect to $t$ for $k_{1}=0.7$ in the case of Solok City 
Figures 3 and 4 also show that the percentage of adult mosquito culling through fogging generally has an effect on decreasing the number of adult mosquitoes. There was a difference in the time of descent to the point of stability between $k_{2}=0$ and $k_{2} \neq 0$, both for the cases of Pekanbaru and Solok.

For the city of Pekanbaru, the decrease in the number of adult mosquitoes generally occurred at $t$ for $k_{2}=0 ; 0,3 ; 0,7 ; 1,0$, meanwhile, for the city of Solok, the number of adult mosquitoes decrease dramatically at $k_{2}=0,3 ; 0,7 ; 1,0$.

In addition to the effect on the number of adult mosquitoes, the eradication of adult mosquitoes also has an effect on the number of mosquitoes that have dengue fever.

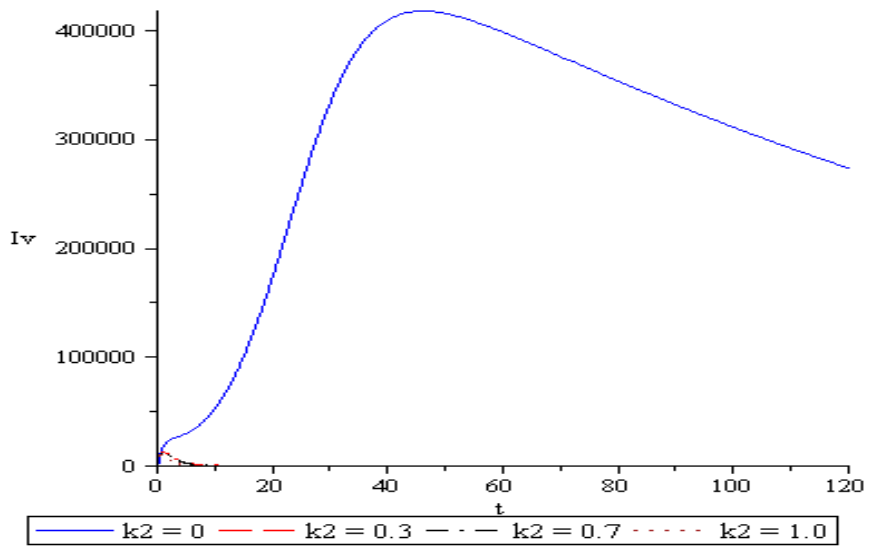

Figure 5. The graph of $I_{v}$ with respect to $t$ for $k_{1}=0.7$ in the case of Pekanbaru City

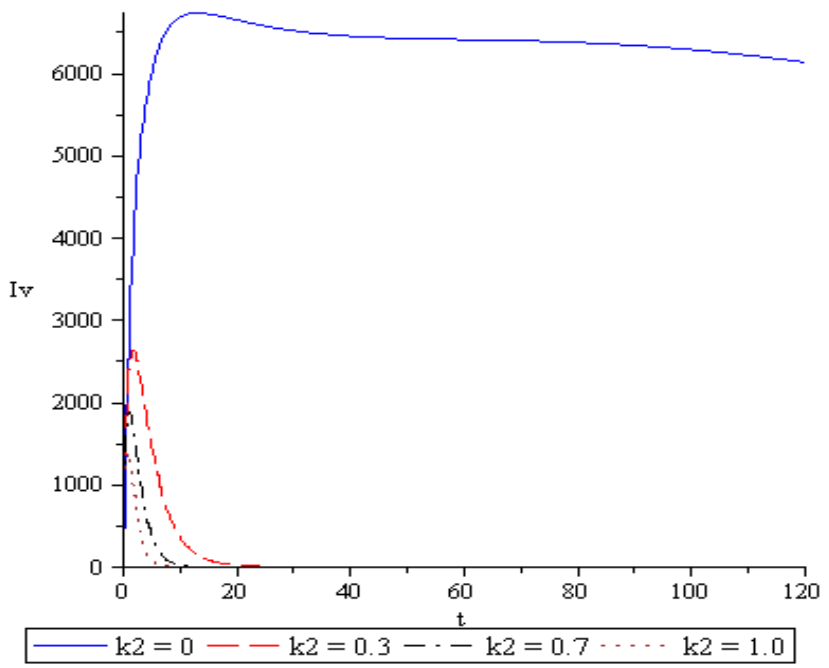

Figure 6. The graph of $I_{v}$ with respect to $t$ for $k_{1}=0.7$ in the case of Solok City

Based on Figures 5 and 6, it shows that if there are no steps to eradicate mosquitoes through fogging, the number of mosquitoes that cause dengue fever (mosquitoes infected with dengue virus) tends to be constant at all times, both in Pekanbaru and Solok.

Furthermore, mosquito eradication can also have an indirect effect on the number of people affected by DHF, as shown in Figures 7 and 8. 


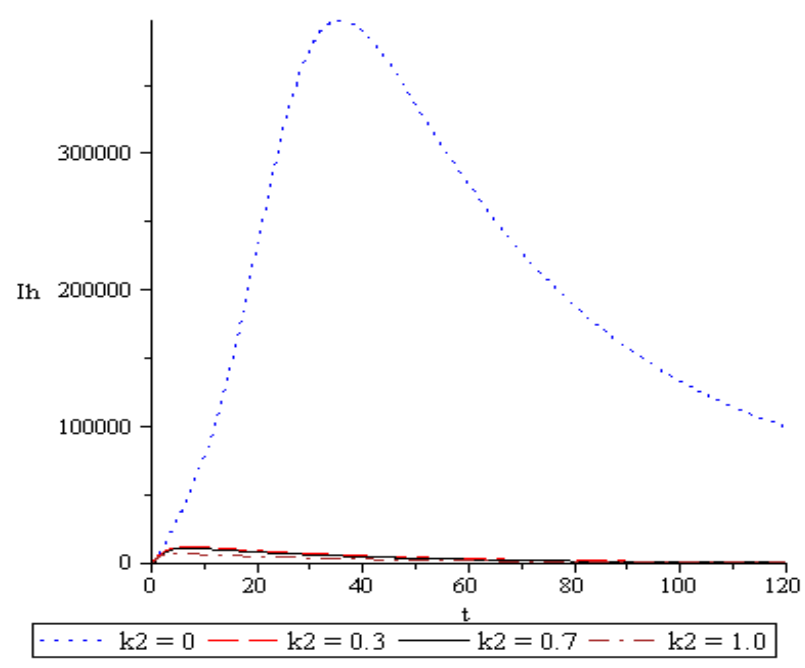

Figure 7. The graph of $I_{h}$ with respect to $t$ for $k_{1}=0.7$ in the case of Pekanbaru City

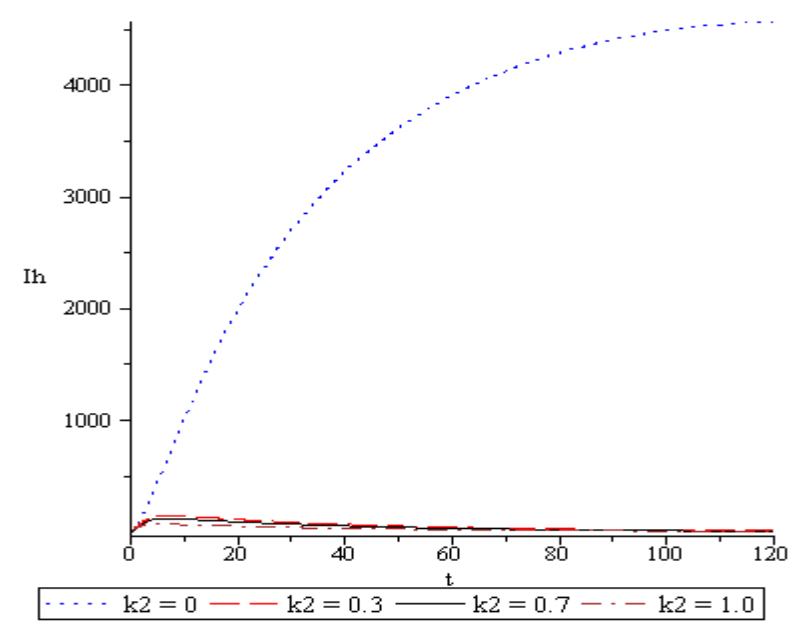

Figure 8. The graph of $I_{h}$ with respect to $t$ for $k_{1}=0.7$ in the case of Solok City

Based on Figures 7 and 8, it can be seen that the eradication of adult mosquitoes has a very significant effect on reducing the number of sick people, both in Pekanbaru and Solok. On the other hand, if mosquito eradication treatment is not given, DHF sufferers tend to increase in Solok city, but tend to decline slowly in the case of Pekanbaru city.

\section{CONCLUSIONS}

Based on the treatment of larvae and adult mosquito eradication $\left(k_{1}\right.$ and $\left.k_{2}\right)$ in both cities, it shows that there is an influence on the number of infected people. However, the city of Pekanbaru has decreased the number of infected people faster than the city of Solok. This is due to the city of Pekanbaru, which has a relatively high temperature $(T)$ compared to the city of Solok.

\section{ACKNOWLEDGMENTS}

We gratefully acknowledge LPPM UIN Sultan Syarif Kasim Riau for funding this research.

\section{REFERENCES}

[1] Kemenkes, "Demam Berdarah Dengue," Buletin Jendela Epidemiologi, vol. 2, p. 48, 2010.

[2] M. M. Rossi, L. F. Lopez, and E. Massad, "The Dynamics of Temperature- And Rainfall-Dependent Dengue Transmission in Tropical Regions," Annals of Biometrics \& Biostatistics, vol. 2, no. 2, pp. 1-6, 2015. 
[3] A. Sumi, E. F. O. Telan, H. Chagan-Yasutan, M. B. Piolo, T. Hattori, and N. Kobayashi, "Effect of temperature, relative humidity and rainfall on dengue fever and leptospirosis infections in Manila, the Philippines," Epidemiology and Infection, vol. 145, no. 1, pp. 78-86, 2017, doi: 10.1017/S095026881600203X.

[4] A. K. Supriatna and E. Soewono, "Model Matematika Penyebaran Penyakit Demam Berdarah," Jurnal Bionatura, vol. 2 , no. 3, pp. 1-13, 2000.

[5] S. Side and S. M. Noorani, "A SIR model for spread of dengue fever disease (simulation for South Sulawesi, Indonesia and Selangor, Malaysia)," World Journal of Modelling and Simulation, vol. 9, no. 2, pp. 96-105, 2013.

[6] S. C. Chen and M. H. Hsieh, "Modeling the transmission dynamics of dengue fever: Implications of temperature effects," Science of the Total Environment, vol. 431, pp. 385-391, 2012, doi: 10.1016/j.scitotenv.2012.05.012.

[7] G. R. Phaijoo and D. B. Gurung, "Modeling Impact of Temperature and Human Movement on the Persistence of Dengue Disease," Computational and Mathematical Methods in Medicine, vol. 2017, 2017, doi: 10.1155/2017/1747134.

[8] R. Taghikhani and A. B. Gumel, "Mathematics of dengue transmission dynamics: Roles of vector vertical transmission and temperature fluctuations," Infectious Disease Modelling, vol. 3, pp. 266-292, 2018, doi: 10.1016/j.idm.2018.09.003.

[9] P. S. Murdoch, J. S. Baron, and T. L. Miller, "Potential Effects of Climate Change on Surface - Water," vol. 36, no. 2, p. 2018, 2018.

[10] P. Chanprasopchai, P. Pongsumpun, and I. M. Tang, "Effect of Rainfall for the Dynamical Transmission Model of the Dengue Disease in Thailand," Computational and Mathematical Methods in Medicine, vol. 2017, 2017, doi: 10.1155/2017/2541862.

[11] A. Bustamam, D. Aldila, and A. Yuwanda, "Understanding Dengue Control for Short- and Long-Term Intervention with a Mathematical Model Approach," Journal of Applied Mathematics, vol. 2018, 2018, doi: 10.1155/2018/9674138.

[12] Y. H. Cheng et al., "Assessing health burden risk and control effect on dengue fever infection in the southern region of Taiwan," Infection and Drug Resistance, vol. 11, pp. 1423-1435, 2018, doi: 10.2147/IDR.S169820.

[13] K. Chudej and A. Fischer, "Optimal Vaccination Strategies for a new Dengue Model with two Serotypes," IFAC-PapersOnLine, vol. 51, no. 2, pp. 13-18, 2018, doi: 10.1016/j.ifacol.2018.03.003.

[14] L. F. Nie and Y. N. Xue, "The roles of maturation delay and vaccination on the spread of Dengue virus and optimal control," Advances in Difference Equations, vol. 2017, no. 1, 2017, doi: 10.1186/s13662-017-1323-y.

[15] C. Schreppel and K. Chudej, "Numerical optimal control applied to an epidemiological model," IFAC-Papers On Line, vol. 51, no. 2, pp. 1-6, 2018, doi: 10.1016/j.ifacol.2018.03.001. 